\title{
STATIC MODELING OF THE DIMENSIONAL CHAINS OF THE HOLE CENTER-FINDERS
}

\author{
Vyacheslav Porokhin $^{1^{*}}$ Yuri Karakulev $^{1}$ Ramil Minnigazimov ${ }^{1}$ Sergey Mednikov ${ }^{1}$ Anvar Zakirov $^{1}$ \\ ${ }^{1}$ Saint Petersburg National Research University of Information Technologies, Mechanics and Optics, \\ Saint Petersburg, Russia
}

The article is devoted to estimate the center-finders accuracy for holes of large-sized case details. Methodology for analyzing center-finders accuracy using dimensional chains and computational mathematics is proposed. It is shown that the quality classes calculated for a direct task can be extended. The methodology is applied to estimate the accuracy of center-finders with typical construction using the example of the specified tolerances for the deviation from alignment in the turbine K-300-240 and the diesel engine 10D100. As a result of calculating the dimensional chain for a direct task, the following values are obtained: 5 quality class for the first case, and 6 for the second case. To check for the inverse task, a computer program that calculates the closing link by the Monte Carlo method with Gaussian distribution is written. Based on the mutual influence of the center-finder supports, tolerances can be extended to 6 and 7 respectively, without accuracy loss.

Key words: Center-finder, Dimensional chain, Monte Carlo method, Mathematical modeling

\section{INTRODUCTION}

One of the main properties that ensure the quality of engineering objects is the geometric accuracy of body parts, such as cylinder blocks, reducer housings, shaft supports and others. These are basic, backbone parts that ensure the working position of other parts, and hence the reliability and longevity of the entire facility.

The functional parameters of mentioned parts are alignment, perpendicularity, parallelism and intersection of the holes axes. To monitor these parameters for largesized parts (more than 8 meters), a sighting method is usually used, which is realized with the aid of a coordinate optical measuring device and a position indicator of the hole center of the detail-the center-finder. If the measuring instruments, as a rule, are universal devices (sighting measuring tubes, digital autocollimators), the center-finders are original devices. By now, there are many models as a finished device and as engineering development. But, despite all their diversity, the task of creating a universal center-finder is not solved. It requires constructive solutions that have new functional properties, the principle of operation, the materials used, the various dimensions that will ensure the given accuracy of fixing the hole center, and hence the accuracy of the control of the base parts [01].

Therefore, when designing new models or analyzing known designs of center-finders, it is necessary to evaluate the error of determining of the hole center.

In this paper, we show the possibility of mathematical modeling using dimensional chain methods, Monte Carlo and computational mathematics for estimating the accuracy of center-finders. Based on these methods, an algorithm is constructed and a numerical modeling of typical center-finders is performed for a straight line (determining the quality class of the constituent links) and the inverse (determining the nominal size of the closing link) tasks.

\section{RESEARCH FACILITY}

To develop a mathematical model and carry out calculations, a typical scheme of the center-finder is chosen (Figure 1, a). It consists of a target mark 1 (grid with a crosshair or a coordinate photodetector), a rotary disk 2 divided into three sectors with a variable profile, and three supports 3 . When the disk is turned, the supports are displaced before contact with the wall of the hole. As a result, the target mark is fixed in the hole center [02].

\section{RESEARCH METODOLOGY}

The actual position of the target mark in the hole is ensured by the accuracy of the center-finder details and their matching. Therefore, when assessing accuracy, it is necessary to consider the interrelated dimensions, their quality classes, deviations, mutual influence on each other. The above tasks are solved using the theory of dimensional chains (DC).

$D C$ of the center-finder is made for one support (Figure $1, b)$. For other supports, because of the symmetry of they construction, the DC's are similar. It is a set of links, where $\mathrm{Ai}$ are the quality classes of the mating parts, $\mathrm{A}_{\Delta}$ - the deviation of the target from the center of the hole.

The DC of the center-finder support allows to solve two problems. At first, to calculate the quality classes and to establish the quality classes of the constituent links based on the specified accuracy requirements for the closing link. Secondly, to set the nominal value and quality class of the closing link according to the established quality classes of the component links [03]. 


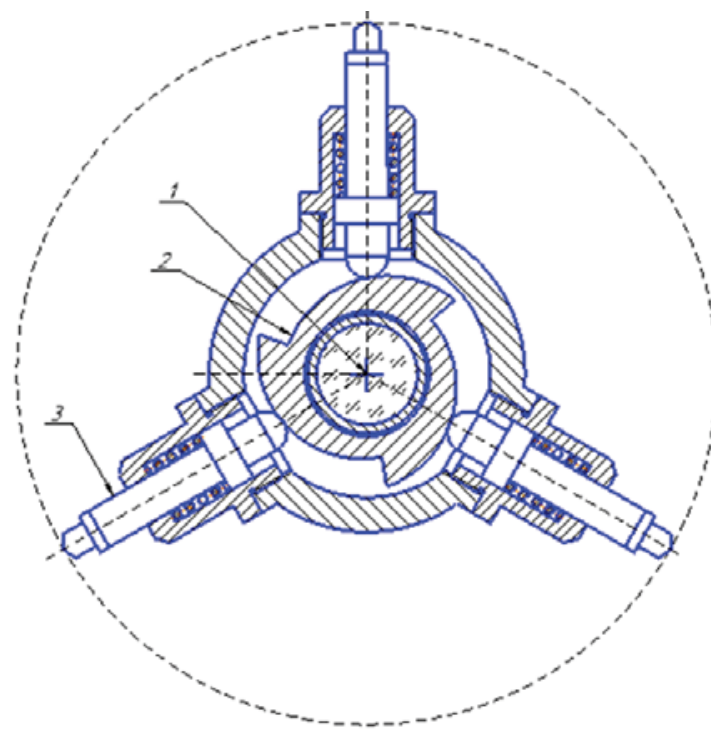

a)

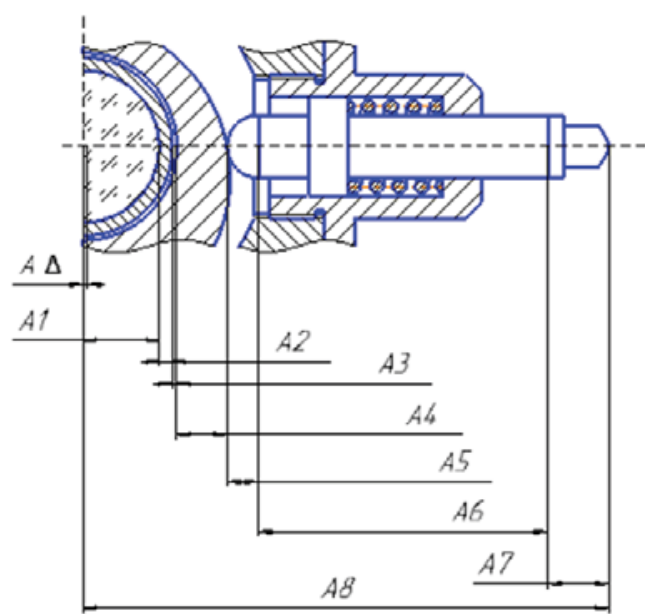

b)

Figure 1: To explain the structure of the center-finder: a) typical scheme; b) dimensional chain of support

Proceeding from the fact that the nominal dimensions included in the RC have values of different orders, the calculations are performed by the "one quality class» way. Required quality class $\mathrm{a}_{\text {avg }}$ is determined by the formula 1:

$$
\mathrm{a}_{a v g}=\frac{\mathrm{T}_{\Delta}}{\sum_{j=1}^{k-1} i_{j}}
$$

where $T_{\Delta}$ is the specified tolerance of the closing link, $i=0.45 \sqrt[3]{D}+0.001 D$ is the tolerance unit, $D$ is the average geometric dimension for the diameter interval to which the given linear dimension belongs [04].

In the verification calculation (inverse task), the parameters of the closing link are determined by the following formulas.

Nominal size of the closing link

$$
\mathrm{A}_{\Delta}=\sum_{i=1}^{n} A_{i}=\sum_{j=1}^{n_{j}} A_{j}-\sum_{q=1}^{n_{q}} A_{q}
$$

Upper deflection of the closing link:

$$
\Delta_{B_{\Delta}}=\Delta_{0_{\Delta}}+\frac{\delta_{\Delta}}{2}
$$

Lower deflection of the closing link:

$$
\Delta_{H_{\Delta}}=\Delta_{0_{\Delta}}-\frac{\delta_{\Delta}}{2}
$$

where $A_{\Delta}$ is the nominal size of the closing link, $A_{i}$ - the nominal size of any component, $A_{-}$-the nominal size of the magnifying unit, $A_{q}-$ the nominal size of the reducing link, $n_{j}$ and $n_{q}$ are the number of increasing and decreasing links, $\Delta_{B \Delta}$ and $\Delta_{H \Delta}$ - upper and lower deviations of the closing link, $\Delta_{O \Delta}$ is the coordinate of the tolerance field middle for the closing link, $\delta_{\Delta}$ is the tolerance of the closing link.

Comparison of the calculated value of the closing link with the specified tolerance will be an inadequate condition for the suitability of the device with these quality classes since, the total deviation will be affected by the symmetry of the structure with respect to the center. In this case each support will affect the position of the target mark relative to the center of the hole as a function of the deviation value. To determine the value of the resulting deviation $\vec{V}$, it is necessary to perform algebraic summation of the deviations along the axes of the center-finder supports - the vectors $\overrightarrow{\mathrm{V} 1}, \overrightarrow{\mathrm{V} 2}, \overrightarrow{\mathrm{V} 3}$ (Figure 2).

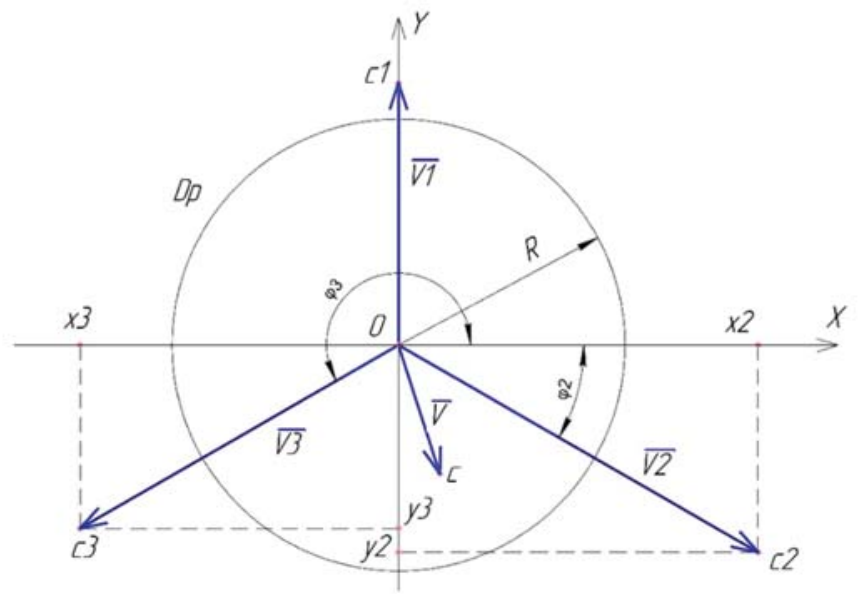

Figure 2: The scheme for finding the resulting deviation 
The coordinates of the vector $\vec{V}$ vertex will be determined by the following formulas:

$$
\begin{aligned}
& c_{x}=\cos (\varphi 2) z v 2+\cos (\varphi 3) z v 3 \\
& c_{y}=z v 1+\sin (\varphi 2) z v 2+\sin (\varphi 3) z v 3
\end{aligned}
$$

where $\varphi 2-$ angle between the axis $\mathrm{OX}$ and the vector $\overrightarrow{V 2} ; \varphi 3$ - angle between the axis $\mathrm{OX}$ and the vector $\overrightarrow{V 3}$ $z v 1, z v 2$ and $z v 3$ are the lengths of the vectors $\overrightarrow{V 1}, \overrightarrow{V 2}$ and $\overrightarrow{V 3} ; D p$ - circle that determines the given tolerance for the accuracy of the center-finder, and the radius $R$ is the tolerance value.

The condition for observing a given accuracy is the fulfillment of inequality:

$$
c_{x}^{2}+c_{y}^{2} \leq R^{2}
$$

In the real device, the dimensions of the constituent links are random variables. Consequently, the terminating link of the DC is also a random variable. Therefore, it is possible to use the statistical modeling method (Monte Carlo) in DC calculations, which is the closest to the real model. It is based on modeling random values of links nominal sizes distributed in the field of their tolerance according to a given law and with given probabilistic characteristics. Passing from a random combination of errors to a random combination of the constituent links sizes, find the i-th realization of the resulting error [05].

When modeling the values of the DC constituent links, the most universal law of scattering of random variables-the normal (Gauss) law is used. At the same time, the proposed mathematical model makes it possible to use other distribution functions [06].

Based on the above, an algorithm and a computer program for calculating the DC are developed. Figure 3 shows the scheme of the program for calculating the closing link of the DC using the Monte Carlo method [07]. The algorithm is constructed as follows. At the beginning, the data are entered: A [...] - an array of nominal values of the links; ES [...] - the upper deviation of the links; EI $[\ldots]$ is a lower deviation; Z [...] - an array for coefficients that determine the type of links; S [...] - an array of values distributed according to the Gaussian law with mathematical expectation equal to 0 , and an standard deviation equal to $1 ; Z V[\ldots]$ - the array of nominal values of the closing link; SZ - mean value of the closing link (deviation of the target sign of the center-finder relative to the center of the monitored hole along the axis of the support).

The array Ai [...] [...] is filled according to the formula for determining the random values of the link in the dimensional chain for a normal truncated distribution. The variables Tv and Tn are the quantiles of the distribution for the one-sided confidence probabilities $Y v$ and $Y n$ corresponding to the upper and lower bounds of the size scattering (for the calculation of $Y v$ and $Y n$ are chosen equal to 0.99865 , respectively, $\mathrm{Tv}$ and $\mathrm{Tn}=3$ ) [08]. Filling of the array $\mathrm{ZV}[\ldots]$ is done by the formula (2).
The value of the variable $n$, which determines the number of values for each link of the RC, is determined by the necessary accuracy of calculations and can vary depending on the computing power of the computer.

The values from the array $\mathrm{ZV}$ [...] are used to solve the Equations 3 and 4 as the lengths of the vectors $z v 1, z v 2$ and $z v 3$, in order to test the suitability of devices whose details are performed according to the calculated quality classes, it is necessary to check the worst case when one closing link corresponds to the max value from array ZV [...], the other to min value, the third to their average value. If under the given conditions inequality (5) is satisfied, then $100 \%$ of the center-finders will meet the specified accuracy requirement.

\section{RESULTS OF THE RESEARCH}

As source data, the tolerances for the location of the real large objects holes were chosen: the turbine of grade K-300-240-1 (the allowed deviation of the bores under the bearing housings is no more than $0.10 \mathrm{~mm}$ ) and the diesel engine of grade 10D100 (permissible deviation of the bearings supports - not more than $0.05 \mathrm{~mm}$ ) [09, 10]. According to formula (1), the value of the aavg $=10.03$ is calculated for the tolerance of $0.10 \mathrm{~mm}$, which corresponds to the 6 th quality class. A similar calculation was carried out with a tolerance of $0.05 \mathrm{~mm}$, from which it follows that the constituent links must be made with 5 quality class. The value of the closing link of the DC is determined according to the calculated qualifications using the developed program.

Calculation, based on the mutual influence of the center-finder supports, shows that the inequality (5) is satisfied for a tolerance of $0.05 \mathrm{~mm}$ at 6 quality class, and for a tolerance of $0.10 \mathrm{~mm}$, at 7 quality class, respectively. The results are shown in Table 1.

Table 1: Results of calculations

\begin{tabular}{|c|c|c|c|}
\hline \multicolumn{2}{|c|}{ Test device } & $\begin{array}{c}\text { Diesel } \\
\text { engine } \\
\text { 10D100 }\end{array}$ & $\begin{array}{c}\text { Turbine } \\
\text { K-300- } \\
240-1\end{array}$ \\
\hline \multicolumn{2}{|c|}{ Specified tolerance, mm } & 0.05 & 0.10 \\
\hline $\begin{array}{c}\text { For the } \\
\text { Constituent } \\
\text { Units }\end{array}$ & $\begin{array}{c}\text { Established } \\
\text { quality class }\end{array}$ & 5 & 6 \\
\hline \multirow{2}{*}{$\begin{array}{c}\text { For the } \\
\text { closing link }\end{array}$} & $\begin{array}{c}\text { Average } \\
\text { value, mm }\end{array}$ & 0.034 & 0.051 \\
\cline { 2 - 4 } & $\begin{array}{c}\text { Upper } \\
\text { deviation, } \\
\text { mm }\end{array}$ & +0.014 & +0.025 \\
\cline { 2 - 4 } & $\begin{array}{c}\text { Lower } \\
\text { deviation, } \\
\text { mm }\end{array}$ & -0.014 & -0.025 \\
\hline \multicolumn{2}{|c|}{$\begin{array}{c}\text { The value of the quality class } \\
\text { based on the total deviation }\end{array}$} & 6 & 7 \\
\hline
\end{tabular}




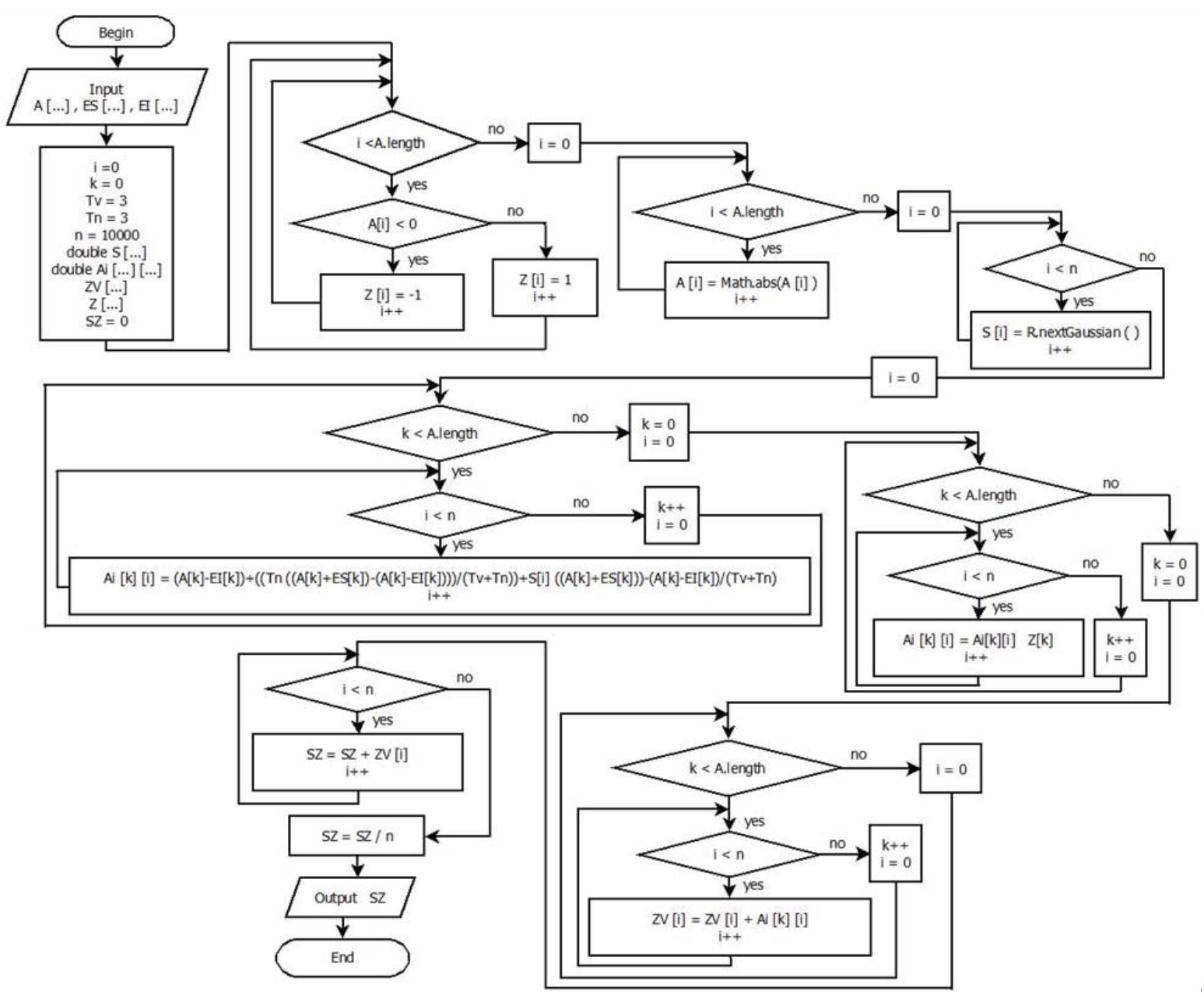

Figure 3: Scheme of the program for calculating the closing link of the DC by the Monte Carlo method

\section{CONCLUSION}

A mathematical model that makes it possible to estimate the accuracy of various designs of the center-finders of the details holes and also to optimize the tolerances for the size of the details of the center-finders by an analytical calculation method is developed. The algorithm and the program for calculating the closing link of the RC by the Monte Carlo method are compiled. Computational research of the mathematical model on real details have shown the efficiency of its use to estimate the accuracy of center-finders.

\section{REFERENCES}

1. State standart $2.308-2011$. Indications of the tolerances of the shape and arrangement of surfaces. (2012). Moscow: Standartinform Publ. (In Russian).

2. Tabakov S.V. Postovalova A.A., (2009). Engineering geodesy. Khabarovsk: Publishing house of the FESTU. (In Russian).

3. RD 50-635-87. Methodical instructions. Chains are dimensional. Basic concepts. Methods for calculating linear and angular chains. (1987). Moscow: Publishing Standards. (In Russian).

4. Putyato A.V. Kovalenko A.V., (2008). Calculation of di- mensional chains. Gomel: BelGUT Publ. (In Russian).

5. Mikhailov G.A. Voitishchev A.V., (2006). Numerical static modeling. Monte Carlo methods. Moscow: Publishing Center "Academy". (In Russian).

6. Valetov V.A. Murashko V.A., (2006). Fundamentals of Instrument Technology. St. Petersburg: SPbNRU ITMO. (In Russian).

7. Gavrilov A.V. Klimenkov S.V. Tsopa E.A., (2010). Programming in Java: Lecture notes. SPb: SPbNRU ITMO. (In Russian).

8. Palei M.A. Romanov A.B. Braginsky V.A. and others, (2001). Tolerances and fits. SPb: Politechnica Publ. (In Russian).

9. Altuhov V. YA., (1989). Mechanization and automation of maintenance and repair of rolling stock. Moscow: Transport Publ. (In Russian).

10. Steam turbine K-300-240-1 LMZ. Technical conditions for overhaul. Norms and requirements. (2009). Moscow. (In Russian).

Paper submitted: 05.06.2017.

Paper accepted: 27.11.2017.

This is an open access article distributed under the CC BY-NC-ND 4.0 terms and conditions. 